\section{The author replies.}

I want to begin by thanking $\mathrm{Dr}$. Benneyan for his careful critique and additional caveats. Further, I will concede many of the points he raises. However, I want to question two of his comments: that "the appropriate situations in which $p, u$, and $c$ charts should be used ... are not dependent on the incident rate," and that control charts should not be viewed as the mere superimposition of control limits on top of a preexistent run chart.

Of course, the probability distribution for the data is theoretically the correct criterion for choosing the type of chart. ${ }^{1-3}$ However, Benneyan himself describes on page 208 of his recent overview of statistical quality control methods how "as the binomial subgroup size $n$ converges to infinity, any binomial distribution converges to a Poisson distribution." 2 I found it instructive that the problem rates in the data he used to illustrate the $p$ chart (his Figure 3 ) and $u$ chart (see top of right column on page 208) conform with the rates (actually rules of thumb) suggested in my Table $1 .{ }^{4}$ Further, as the denominator for data suitable for a $u$ chart increases, the $c$ chart can be shown empirically to give a good approximation to the result one would get from the $u$ chart. Not that Dr. Benneyan encourages the use of approximate methods; he most certainly does not, even while acknowledging the similarity of results that may be found when using them.,3 For the novice users to whom my caveats were addressed, users who do not think of data in terms of probability distributions, approximate methods and rules of thumb may be very useful. It is important to remember that not all those charged with producing control charts bring to the task the same statistical skills; compare, for example, the statistical backgrounds of a typical quality manager in a healthcare setting with those of a typical quality engineer.

I take the point that, for charts that do not simply plot raw data (eg, charts designed to deal with auto-correlated data or risk-adjusted values), control charts are not constructed by "superimposing control limits on top of a preexistent run chart." However, for the simpler $p, c$, and $u$ charts, all other teachers in my experience have held that the only difference in the run and control charts is the addition of said limits and the associated A, B, and $C$ zones. My primary intention in this section of the text was not to make the distinction between run and control charts. I was encouraging novice users to resist the urge (either from within or from others) to construct control charts before they have amassed the recommended number of data points. This is obviously something that Dr. Benneyan feels deeply about, given that in his overview he states three times that at least 25 data points are needed. ${ }^{2}$ I was proposing the use of run charts in the interim.

Again, I want to thank Dr. Benneyan for his comments and for his recent overview of the theory and practice of control charting. ${ }^{2,3} \mathrm{Had}$ it appeared before I submitted the final draft of my article, I would have included it among the sources I recommended for those interested in learning more about these important and deceptively complex tools. ${ }^{4}$

\section{REFERENCES}

1. Sellick JA Jr. The use of statistical process control charts in hospital epidemiology. Infect Control Hosp Epidemiol 1993;14:649-656.

2. Benneyan JC. Statistical quality control methods in infection control and hospital epidemiology, part I: introduction and basic theory. Infect Control Hosp Epidemiol 1998;19:194-214.

3. Benneyan JC. Statistical quality control methods in infections control and hospital epidemiology, part II: chart use, statistical properties, and research issues. Infect Control Hosp Epidemiol 1998;19:265-277.

4. Humble C. Caveats regarding the use of control charts. Infect Control Hosp Epidemiol 1998;19:865-868.

Charles Humble, PhD

National Performance Data Feedback Center

Durham, North Carolina

\section{Bugs Are Not Funny Syndrome}

\section{To the Editor:}

The recent identification of vancomycin-intermediate Staphylococcus aureus has reinvigorated legitimate fears in the infection control commu- nity that the microbe world is going amuck. This has had a dramatic impact on the behavior of a few of our fellow infection control professionals. Signs and symptoms include the following:

1. Contemplating a telephone condom for your office;

2. Taking a shower with a mask on to prevent legionnaire's disease;

3. Installing sinks at the front door so guests can wash before entering;

4. Washing your hands before and after going to the bathroom, just in case;

5. Demanding that waiters wash their hands before serving meals (and observing them do so);

6. Keeping a bottle of disinfectant spray handy to use on hand-held items between use;

7. Removing all blenders from your home;

8. Always carrying an extra pen in case someone asks to borrow one, so you can say, "keep it, I have an extra";

9. Requiring sedation when someone in an elevator sneezes without covering his or her mouth;

10. Always being prepared by carrying a "sinkless" handwashing agent wherever you go;

11. Placing handwashing stickers in your home bathroom as a reminder for family members and guests to wash up;

12. Waking up in a cold sweat from a nightmare involving mouth pipetting, eating, and drinking in the laboratory;

13. Development of rage attacks when making rounds and seeing a patient in Contact Precautions for methicillin-resistant coagulasenegative staphylococci.

Steven J. Schweon, RN, MPH Ellen Novatnack, RN, BSN St Luke's Hospital Bethlehem, Pennsylvania

Eileen O'Rourke, MT(ASCP), CIC Pennsylvania Hospital

Philadelphia, Pennsylvania

Susan Trout, RN, CIC Montgomery Hospital Norristown, Pennsylvania 